\title{
Nonalcoholic Fatty Liver Disease and Cardiovascular Disease: Has the Time Come for Cardiologists to Be Hepatologists?
}

\author{
Mohamed H. Ahmed, ${ }^{1}$ Salma Barakat, ${ }^{2}$ and Ahmed O. Almobarak ${ }^{3}$ \\ ${ }^{1}$ Department of Medicine, Wexham Park Hospital, Berkshire, Slough, UK \\ ${ }^{2}$ National Center for GI \& Liver Diseases, Ibn Sina Hospital, Ministry of Health, Khartoum, Sudan \\ ${ }^{3}$ Department of Pathology, Faculty of Medicine, University of Medical Sciences and Technology, \\ Khartoum, Sudan \\ Correspondence should be addressed to Mohamed H. Ahmed, elziber@yahoo.com
}

Received 23 October 2012; Accepted 23 November 2012

Academic Editor: Mahir A. Hamad

Copyright (C) 2012 Mohamed H. Ahmed et al. This is an open access article distributed under the Creative Commons Attribution License, which permits unrestricted use, distribution, and reproduction in any medium, provided the original work is properly cited.

\begin{abstract}
Nonalcoholic fatty liver disease (NAFLD) is prevalent in people with the metabolic syndrome and type 2 diabetes and is present in up to one-third of the general population. Evidence is now accumulating that NAFLD is associated with obesity and diabetes and may serve as a predictor of cardiovascular disease (CVD). The possible mechanisms linking NAFLD and CVD include inflammation and oxidative stress, hyperlipidaemia, insulin resistance, and direct impact of NAFLD on coronary arteries and left ventricular dysfunction. In addition, several studies suggest that NAFLD is associated with high risk of CVD and atherosclerosis such as carotid artery wall thickness and lower endothelial flow-mediated vasodilation independently of classical risk factors and components of the metabolic syndrome. It is not yet clear how treatment of NAFLD will modulate the risk of CVD. Furthermore, studies are urgently needed to establish (i) the pathophysiology of CVD with NAFLD and (ii) the benefit of early diagnosis and treatment of CVD in patients with NAFLD. In the absence of biochemical markers, it is crucial that screening and surveillance strategies are adopted in clinical practice in the growing number of patients with NAFLD and at risk of developing CVD. Importantly, the current evidence suggest that statins are safe and effective treatment for CVD in individuals with NAFLD.
\end{abstract}

\section{Introduction}

Nonalcoholic fatty liver disease (NAFLD) is emerging as an important public health problem across the globe and associated with high risk for cardiovascular disease (CVD) for adult and children. NAFLD refers to a wide spectrum of liver damage, ranging from simple steatosis to steatohepatitis, advanced fibrosis, and cirrhosis. NAFLD is strongly associated with insulin resistance and is defined by the accumulation of liver fat $>5 \%$ per liver weight, in the presence of $<10 \mathrm{~g}$ of daily alcohol consumption. NAFLD is associated with type 2 diabetes, obesity, and hyperlipidemia $[1,2]$. The prevalence of NAFLD is estimated to be around $34 \%$ of total population, while obesity with nonalcoholic fatty liver disease varies between $30 \%$ and $100 \%$, whereas the prevalence of NAFLD within type 2 diabetes varies between 10 and $75 \%$.
Interestingly, the prevalence of NAFLD in USA is around $34 \%$ and $90 \%$ of these cases of NAFLD were attributed to nonalcoholic causes $[3,4]$. In the Dionysos nutrition and liver disease study in Italy, we found that the prevalence of NAFLD was $\sim 25 \%$ and was associated with most features of the metabolic syndrome [5]. Interestingly, alcohol intake and obesity could both act in a synergistic way to increase risk of in mortality. For instance, the One Million Women Study showed that women who are obese or overweight had an increased risk of liver cirrhosis. The absolute risk of liver cirrhosis with obesity has also been shown to be increased synergistically with only $\sim 15$ units of alcohol per week [6]. Furthermore, Hart et al. found that obesity and alcohol (consuming 15 or more units per week) intake are associated with greater risk of dying of liver disease [7]. It is plausible to predict that, with an increase in prevalence of epidemic of diabetes and obesity, it is likely that there will 
be a marked increase in prevalent NAFLD with important consequences for health care providers [8]. On the other hand, in nondiabetic and nonobese individuals, insulin resistance, hypertriglyceridemia and hyperuricemia were independently associated with NAFLD [9]. Importantly, the increase in BMI is associated with higher prevalence of NAFLD. The prevalence of NAFLD increased from $16.4 \%$ among individuals with normal BMI to $75.8 \%$ among obese people. The prevalence of NAFLD is even higher with morbid obesity, and among morbidly obese undergoing bariatric surgery the prevalence may be as high as 96\% [10]. The prevalence of NAFLD showed different pattern in different ethnic groups. For instance, in Japan the prevalence of NAFLD was $25 \%$ and in China $24 \%$, while in a small study in diabetic Saudi individuals have suggested that the prevalence of NAFLD is $\sim 55 \%$ [11-14]. Children are also known to be in risk of developing NAFLD. In a retrospective review of 742 children between the ages of 2 and 19 years who had an autopsy performed from 1993 to 2003, fatty liver was present in $13 \%$ of subjects [15]. In a small study of 44 obese children, fatty liver was identified in 14 subjects (31.8\%) [16].

One important concern with silent undiagnosed NAFLD is that it may progress silently and result in cirrhosis, portal hypertension, and liver-related death in early adulthood. Interestingly, NAFLD is the hepatic component of the metabolic syndrome and is an independent risk factor for cardiovascular disease (CVD). Importantly, NAFLD is associated with an increased risk of all-cause death and predicts future CVD events, independently of the classical risk factors like age, sex, LDL cholesterol, smoking, and features of metabolic syndrome [8]. One significant challenge for mass screening for NAFLD is the lack of sensitive and specific biochemical marker. The concomitant use of alanine aminotransferase ALT and ultrasound may produce high diagnostic [17].

NAFLD is associated with an increase in morbidity and mortality. For instance, Adams et al. have studied the impact of NAFLD on mortality among 337 patients with type 2 diabetes followed for 9 years (Adams et al. 2005). In multivariate analysis to adjust for confounders, overall mortality was significantly associated with a diagnosis of NAFLD, presence of ischemic heart disease, and duration of diabetes [18]. Interestingly, fatty liver and smoking had synergistic effect in metabolic syndrome and it is parameters, especially, low HDL-c and high plasma triglyceride [19]. Therefore, individuals with features of the metabolic syndrome, obesity, consume alcohol, and smoking with fatty liver will need aggressive treatment to decrease the risk of CVD.

\section{NAFLD, Metabolic Syndrome, and Diabetes}

A joint interim statement of the International Diabetes Federation Task Force on Epidemiology and Prevention; National Heart, Lung, and Blood Institute; American Heart Association; World Heart Federation; International Atherosclerosis Society; and International Association for the Study of Obesity they define an individual with the metabolic syndrome with three of the five criteria-including elevated waist circumference, elevated triglycerides, and reduced high-density lipoprotein (HDL) — cholesterol levels, elevated blood pressure, and elevated fasting-glucose levels. In this new definition, waist circumference is just one of five criteria that physicians can use when diagnosing the metabolic syndrome (Population-and country-specific definitions) [20]. Insulin resistance which is a landmark of metabolic syndrome has been shown to be associated with $95 \%$ of individuals with NAFLD. Importantly, both metabolic syndrome and NAFLD are associated with high incidence of CVD and most features of metabolic syndrome present in subjects with NAFLD [8].

There is now general agreement in the literature that NAFLD is another clinical feature of the metabolic syndrome $[2,5,8,21]$. A study of 66 patients with NAFLD showed that $98 \%$ were insulin resistant, and $39 \%$ of those were diabetic [22]. In another study, 19 NAFLD patients who were not obese or did not have diabetes and had a normal lipid profile, $47 \%$ showed features of the metabolic syndrome [23]. A marked increase in body mass index (BMI) and waist/hip ratio has been shown in NAFLD patients [24]. Insulin resistance is not dependent on BMI but is related to central obesity, which is a feature of NAFLD. Obesity is associated with a higher incidence of a wide spectrum of liver diseases associated with NAFLD from steatosis to fibrosis and cirrhosis [25]. Recent study carried in 19 European centres showed high association of decreased insulin sensitivity and increased incidence of CVD with NAFLD [26]. Therefore, screening for diabetes is an essential part of the assessment of risk of CVD in individuals with NAFLD.

\section{NAFLD and Its Association with Cardiovascular Disease}

Several studies have shown association of NAFLD with cardiovascular disease. In individuals with diabetes and no diabetes, NAFLD is associated with increased risk of CVD [27, 28]. In a study of biopsy-proven NAFLD with followup for around 21 years the main cause of death in these patients with NAFLD was CVD and malignancy [27]. Interestingly, the histological severity of NAFLD and inflammation is strongly associated with increased risk of CVD and atherogenic lipid profile [28].

In contrast, Domanski et al. 2012 conducted a retrospective chart review of patients with biopsy-confirmed NAFLD: of these, 219 patients had biopsies showing the presence of at least grade I NASH. The overall prevalence of cardiovascular disease was $6.63 \%$ independent of age, sex, body mass index, and the presence of diabetes [29]. On the other hand, simple hepatic steatosis is being shown to be associated with marked silent carotid atherosclerosis [30]. Several cross-sectional studies have reported a relationship between elevated serum activity of liver enzymes (e.g., alanine aminotransferase (ALT) and gamma-glutamyltransferase $(\gamma \mathrm{GT})$ ) and metabolic syndrome, diabetes, and NAFLD. Raised serum activity of liver enzymes independently predicted the future development of metabolic syndrome and DM as well as cardiovascular $(\mathrm{CV})$ events and/or total/CV 
mortality in prospective studies. Importantly, these associations can be partly attributed to nonalcoholic fatty liver disease (NAFLD) and insulin resistance, and there may be additional underlying mechanisms that contribute to the increased CV risk (e.g., inflammation and oxidative stress). The association of $\gamma \mathrm{GT}$ with atherosclerotic plaque is of particular importance in individuals with NAFLD [31, 32]. In a study of 14,874 middle-aged Finnish men and women, mildly increased GGT levels were independently associated with an increased risk of ischemic stroke in both sexes [33]. Among 7,613 middle-aged British men followed for 11.5 years, increased GGT levels were independently associated with a significant increase in mortality from all causes and from CHD [34]. Kozakova et al. showed that serum gammaglutamyltransferase may represent a link between fatty liver and development of early atherosclerosis [35].

Recent epidemiological studies have suggested the increase in ALT may be linked to increase in risk of CVD. The Hoorn Study showed that the association between ALT and CHD events is significant [36], suggesting that NAFLD is associated with CHD independently of other features of the metabolic syndrome. Interestingly, serum ALT is also shown to be positively associated with an increased risk of carotid atherosclerosis in NAFLD [37]. Furthermore, another study examined the association between elevated serum ALT activity and the 10-year risk of CHD as estimated using the Framingham risk score (FRS). The ALT threshold for increased risk of CHD was higher in men ( $>43 \mathrm{IU} / \mathrm{L})$ than in women ( $>30 \mathrm{IU} / \mathrm{L})$ [38]. Recently, the Firenze Bagno a Ripoli (FIBAR) study concluded that increased gamma GT or AST is an independent predictor of CVD. An increase of gamma-GT levels above the reference range, or also in the upper reference, range was also an independent predictor of incident of diabetes [39]. Lopez-Suarez et al. showed in an observational study, the percentage of cases with hypertension was $21.2 \%$ greater in individuals with NAFLD than those without NAFLD (95\% CI, 11.8-30.6, $P<0.0005)$. NAFLD was independently associated with prevalent hypertension with an adjusted odds ratio of 1.71 (95\% CI, 1.10-2.65, $P=0.017$ ). Among nonhypertensive participants, NAFLD was also independently associated with high-normal systolic BP (adjusted odds ratio 2.13, 95\% CI, 1.08-4.20, $P=0.029$ ) but not with high-normal diastolic BP. The authors concluded that the detection of NAFLD, even with normal ALT levels, should serve as an opportunity to identify metabolic and BP abnormalities and intensify lifestyle modification [40].

However, the US Third National Health and Nutrition Examination Survey (NHANES III: 1988-94) study showed that NAFLD was not associated with an increased risk of death from all causes, cardiovascular disease, cancer, or liver disease [41]. Interestingly, data from the National Health and Nutrition Examination Survey III study showed that NAFLD was associated independently with CVD, after adjusting for major demographic, clinical, and metabolic confounders (odds ratio, 1.23; 95\% confidence interval, $1.04-1.44)$. The independent association of NAFLD with cardiovascular mortality was not statistically significant. The authors concluded that NAFLD is associated independently with an increased risk of CVD. However, NAFLD did not increase cardiovascular mortality over a 14 -year period [42].

In individuals with diabetes and NAFLD there is an association between NAFLD and CVD. For instance, data by Targher et al. showed that the unadjusted prevalence of NAFLD was $69.5 \%$ among participants with type 2 diabetes $(n=2839)$, and ultrasound detected NAFLD was the most common cause $(81.5 \%)$ of hepatic steatosis. NAFLD patients had a higher age $(P<0.001)$, and sex-adjusted prevalence of coronary (26.6 versus $18.3 \%$ ), cerebrovascular (20.0 versus $13.3 \%$ ), and peripheral (15.4 versus $10.0 \%$ ) vascular disease than their counterparts without NAFLD. In logistic regression analysis, NAFLD was associated with prevalent CVD independent of classical risk factors, glycaemic control, medications, and the metabolic syndrome features [43]. In addition, the Valpolicella Heart Diabetes Study is a prospective control study in 2,103 type 2 diabetic patients who were free of diagnosed CVD at baseline. During 5 years of followup, 248 participants (case subjects) subsequently developed nonfatal coronary heart disease (myocardial infarction and coronary revascularization procedures), ischemic stroke, or cardiovascular death. After adjustment for age, sex, smoking history, diabetes duration, $\mathrm{HbA}_{1 \mathrm{c}}$, $\mathrm{LDL}$ cholesterol, liver enzymes, and the use of medications, the presence of NAFLD was significantly associated with an increased CVD risk (odds ratio 1.84, 95\% CI 1.4-2.1, $P<$ 0.001). Additional adjustment for the metabolic syndrome (as defined by National Cholesterol Education Program Adult Treatment Panel III criteria) appreciably attenuated, but did not abolish, this association $(1.53,1.1-1.7, P=0.02)$ [44].

Of significant is that NAFLD during childhood is strongly associated with atherosclerosis [45]. In a casecontrol study of 150 overweight children with biopsyproven NAFLD, children with NAFLD had significantly higher fasting glucose, insulin, total cholesterol, low-density lipoprotein cholesterol, triglycerides, systolic blood pressure, and diastolic blood pressure. Subjects with NAFLD also had significantly lower high-density lipoprotein cholesterol than controls. After adjustment for age, sex, race, ethnicity, body mass index, and hyperinsulinemia, children with metabolic syndrome had 5.0 (95\% confidence interval, from 2.6 to 9.7) times the odds of having NAFLD as overweight and obese children without metabolic syndrome. Therefore, NAFLD in overweight and obese children is strongly associated with multiple cardiovascular risk factors. The authors concluded that the identification of NAFLD in a child should prompt global counseling to address nutrition, physical activity, and avoidance of smoking to prevent the development of cardiovascular disease and type 2 diabetes [46].

\section{Possible Pathways and Mechanism of NAFLD Induced CVD}

The association of NAFLD with metabolic syndrome and diabetes may in part explain the increased risk of CVD with NAFLD. In addition, several studies showed that NAFLD in itself might contribute to the increased CVD risk. However, 
the exact mechanisms for this complex relationship are not clear. Probably several highly interrelated factors contribute to the enhanced risk of diabetes and metabolic syndrome in persons with NAFLD. The following factors are possible explanation of mechanisms of the association of CVD with NAFLD (Figures 1 and 2).

4.1. NAFLD May Accelerate the Progression of Insulin Resistance. It is well documented in the literature that NAFLD is associated with an increase in insulin resistance [1-6]. NAFLD is associated with hepatic and peripheral insulin resistance. Importantly, insulin resistance in the pathogenesis of NAFLD and associated with dyslipideamia, carotid atherosclerosis, and endothelial dysfunction.

4.2. NAFLD May Accelerate the Progression of Dyslipidaemia and Atherosclerosis. NAFLD is associated with atherogenic dyslipidaemia (an increase in LDL-c, TG, apolipoprotein B, and decrease in HDL) this associated with an increase in risk of CVD [1-6].

4.3. NAFLD May Accelerate the Progression of Carotid Atherosclerosis. Recent studies show a link between NAFLD and increased carotid intima-media thickness (IMT). Fracanzani et al. (2008) concluded (in a series of normal and NAFLD subjects) that independent risk predictors of increased intima-media thickness were the presence of hepatic steatosis (odds ratio $(\mathrm{OR})=6.9$ ), age $(\mathrm{OR} 6.0)$, and increased systolic blood pressure (OR 2.3) [47]. More interestingly, Targher et al. suggested that the severity of liver histopathology among 85 NAFLD patients is strongly associated with early carotid atherosclerosis, independent of age, sex, BMI, smoking, LDL cholesterol, insulin resistance, and the presence of metabolic syndrome [48]. In addition, a large population study showed that NAFLD is associated with increase in IMT [49]. On the other hand, in 100 dietcontrolled type 2 diabetic individuals, the significant increase of carotid IMT in the presence of NAFLD has largely been explained by insulin resistance. However, Petit et al. showed that in people with type 2 diabetes fatty liver is not associated with increased risk of CVD [50]. In contrast, NAFLD is independently associated with carotid atherosclerosis and only in people with multiple features of the metabolic syndrome [51].

4.4. NAFLD May Accelerate the Progression of Endothelial Dysfunction. Endothelial dysfunction is an important element in the process of atherosclerosis. NAFLD in diabetic and nondiabetic individuals is associated with marked impairment in endothelial function $[52,53]$.

4.5. Inflammation and Oxidative Stress. Inflammation is crucial in pathogenesis of NAFLD, and fat is now considered a metabolically active endocrine organ producing proinflammatory cytokines including TNF- $\alpha$, IL-6, and IL8 and there is an evidence to support the activation of other inflammatory pathways, oxidative stress, and the de novo pathway by TNF- $\alpha$. Inflammation is associated insulin

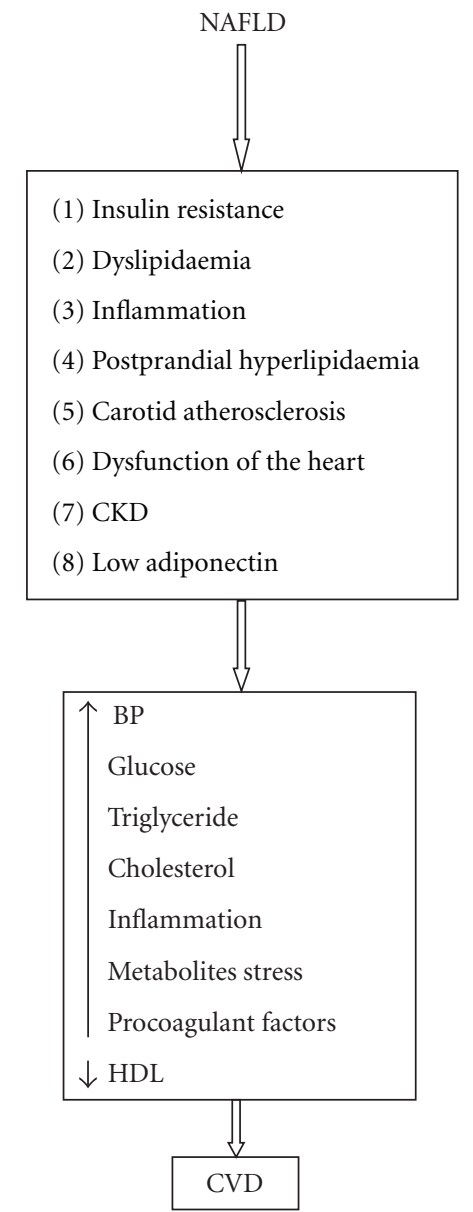

FIGURE 1: It shows possible potential mechanism of the association of NAFLD with CVD.

resistance resulting in increased lipolysis in adipose tissue, increased NEFA uptake by hepatocytes and increased triglyceride synthesis in the liver. Mitochondrial fat oxidation and export of VLDL particles are not able to match triglyceride synthesis leading to net fat deposition in the hepatocytes. As a consequence of abnormal fat accumulation in the hepatocytes, there is a marked derangement in the insulinsignalling pathways in the liver $[54,55]$. Inflammation is the link between obesity and insulin resistance and may have an important role in the pathogenesis of hepatic and systemic insulin resistance and CVD.

4.6. Adiponectin. Adiponectin is one of the important adipocytokines in the pathogenesis of NAFLD. Adiponectinhas been shown to decrease de novo fatty acid synthesis and enhance fat oxidation, with levels of adiponectin increasing after dietary fat ingestion [56]. Decreased adiponectin is associated with insulin resistance and hyperlipidaemia, and low level of adiponectin was shown in NAFLD independent of the components of the metabolic syndrome $[57,58]$. Importantly low levels of adiponectin are also shown to be associated with CVD [59]. 


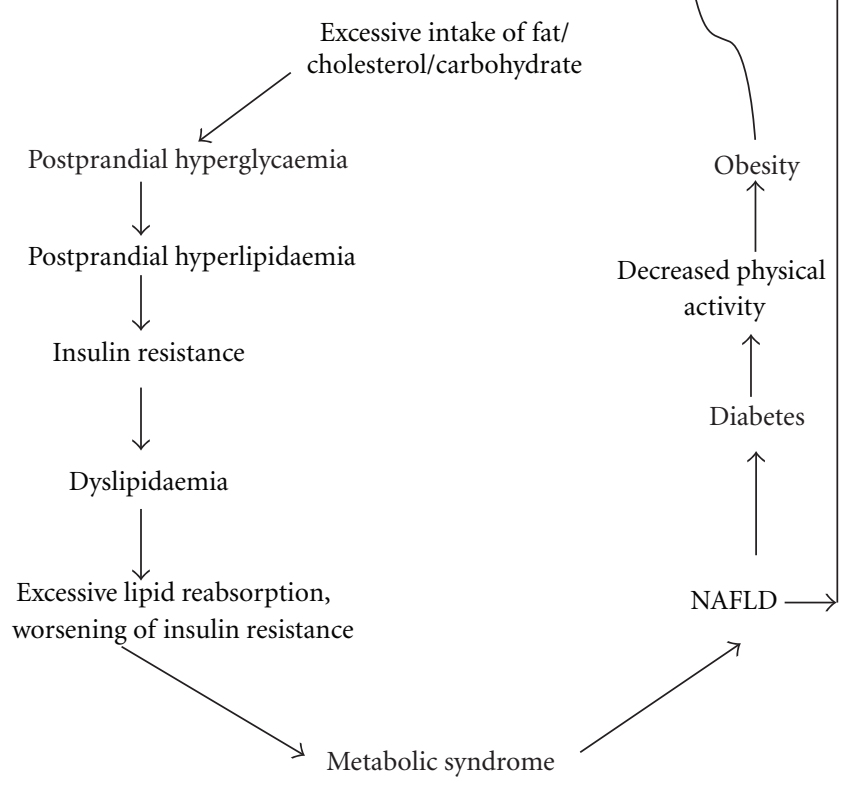

FIgure 2: The metabolic cycle is an attempt to encompass traditional and emerging risk factors of CVD associated with NAFLD in one paradigm. The metabolic cycle is also an attempt to provide possible explanation why complex metabolic factors may lead to development of risk of CVD associated with NAFLD. In addition NAFLD per se can be associated with increased risk of CVD.

4.7. Postprandial Hyperlipidaemia. Postprandial hyperlipidaemia is a risk factor for both NAFLD and CVD. Interestingly, Matikainen et al. suggested that liver fat content is a close correlate of postprandial lipids predicting the responses of triglyceride (TG)-rich lipoproteins (TRL) in chylomicrons and VLDL better than measures of glucose metabolism or body adiposity. High liver fat content associated with postprandial lipemia represents potential risk factors for cardiovascular disease [60]. Furthermore, high dietary fat intake has been shown to induce perturbations in insulin signaling and rates of lipid synthesis via increased hepatic free fatty acid (FFA) flux and triglyceride (TG) [61]. Interestingly, Hadaegh et al. (2008) [62] showed that TG/HDL$\mathrm{C}$ ratio is a independent predictor of coronary heart disease in men. Furthermore, 2 prospective large cohort studies with large numbers of individuals showed clearly that elevated nonfasting triglyceride levels were associated with increased risk of $\mathrm{MI}$, IHD, and death in men and women [63]. In woman nonfasting triglyceride levels were associated with higher cardiovascular events, independent of traditional cardiac risk factors [64]. In addition, nonfasting triglyceride is also been shown to be associated with high risk of ischemic stroke [65]. Generally speaking postprandial lipaemia may emerge as separate CVD risk factor as is being suggested by Mora et al. that nonfasting HDL cholesterol, triglycerides, total/HDL cholesterol ratio, and apolipoprotein A predict CVD better than measured in the fasting state [66]. Therefore, postprandial hyperlipidaemia may in part explain why some lean, overweight, and obese individuals with NAFLD may encounter CVD despite normal fasting lipid profile or being in lipid-lowering medication.

4.8. NAFLD Associated with Disturbance in the Function of the Heart. Several studies have shown the NAFLD is associated with coronary atherosclerosis, heart valve abnormality, and left ventricular dysfunction. For instance, Kim et al. showed that patients with NAFLD are at increased risk for coronary atherosclerosis independent of classical coronary risk factors, including visceral adiposity. The authors claimed that NAFLD per se might be an independent risk factor for coronary artery disease [67]. Furthermore, biopsy-proven NAFLD is associated with marked reduction in coronary flow reserve (marker of the integrity of coronary microvascular circulation). Liver fibrosis scores are found to be independent predictor of marked decrease in coronary flow reserve [68]. In patients with NAFLD the absence of morbid obesity, hypertension, and diabetes has mildly altered LV geometry and early features of left ventricular diastolic dysfunction [69]. In patient with diabetes and NAFLD early features of LV diastolic dysfunction may be detected [70].

4.9. NAFLD and Obstructive Sleep Apnea (OSA). Obstructive sleep apnea (OSA) is characterised by loud and frequent snoring, periods of apnea during sleep, and excessive day somnolence $[71,72]$. Interestingly, OSA is also regarded as one of the factors that accelerate the progression of NAFLD to NASH [73]. Importantly considerable numbers of studies showed an increase incidence of CVD with OSA [74-76]. Animal studies showed that OSA can lead to increase in insulin resistance and alteration in lipid metabolism with NAFLD $[77,78]$. It is possible to suggest that high fat diet in association with OSA may aggravate the course of NAFLD. Furthermore, in a rat model of fatty liver (a choline-deficient high-fat diet) intermittent hypoxia was shown to induce NASH $[79,80]$. The conclusion of Bradley and Floras is that an association between OSA and CVD exists [81]. Their conclusion was that OSA is associated with an increased risk of CVD, and this has been demonstrated in epidemiological, clinical and physiological studies. Epidemiological studies have shown a significant independent association between OSA and hypertension, coronary artery disease, arrhythmias, heart failure, and stroke [82-88]. The complex interaction between obesity, NAFLD, obesity metabolic syndrome, and CVD will accelerate atherosclerosis development.

4.10. NAFLD and CKD That Associated with CVD. NAFLD may also be associated with a detrimental effect on other organs that may have direct or indirect influence on CVD or organs that may accelerate the presentation of CVD, at least in people with type 2 diabetes. For example, NAFLD patients with type 2 diabetes had higher $(P<0.001)$ age- and sexadjusted prevalence rates of both nonproliferative (39 versus $34 \%$ ) and proliferative/laser-treated retinopathy (11 versus $5 \%$ ), and chronic kidney disease (CKD) (15 versus 9\%) than counterparts with type 2 diabetes but without NAFLD [89]. Furthermore, in this study, NAFLD was associated 
with an increased incidence of CKD [90], independent of gender, age, body mass index, waist circumference, BP, smoking, diabetes duration, glycosylated hemoglobin, lipids, baseline estimated GFR, microalbuminuria, and medications (hypoglycemic, lipid-lowering, antihypertensive, or antiplatelet drugs). Interestingly, NAFLD has been shown to be associated with the development of CKD in Korean individuals [91]. Therefore, NAFLD may indirectly modulate the risk of CVD through CKD.

\section{The Safety and Effectiveness of Statins Therapy in NAFLD}

The Practice Guideline by the American Association for the Study of Liver Diseases, American College of Gastroenterology, and the American Gastroenterological Association for diagnosis and management of nonalcoholic fatty liver disease recommended that statins can be used to treat dyslipidaemia in patients with NAFLD and NASH. This recommendation is based on lack of evidence that patients with NAFLD and $\mathrm{NASH}$ are at increased risk for serious drug-induced liver injury from statins [92]. In addition, the Liver Expert Panel stated that statins can be safely used in NAFLD and NASH without the needs for routine liver enzyme monitoring [93]. However, it is recommended before starting statin therapy to check liver enzymens. Statins therapy is shown to improve histology in NAFLD and NASH [94]. Therefore, the current evidence suggest that statin is safe and effective in NAFLD.

\section{Conclusion}

NAFLD is an important, and emerging heath problem. NAFLD is regarded as hepatic component of the metabolic syndrome and associated with high risk of development of CVD. Several studies suggest that NAFLD per se can be associated with risk of CVD. Dyslipidemai, insulin resistance, low adiponectin, and postprandial dyslipidaemai and hyperglycaemia are main factors lead to NAFLD and further aggravate the course of NAFLD as well as accelerate the progress of atherosclerosis and development of CVD. The mechanism and complex factors involved in the development of CVD in individuals with NAFLD are summarised in Figures 1 and 2. Currently, it is not known how treatment of NAFLD will modulate the risk of CVD. Further research is urgently needed to understand the association between NAFLD and CVD and how potential treatment of NAFLD will modulate the risk of CVD.

\section{References}

[1] M. H. Ahmed and C. D. Byrne, "Obstructive sleep apnea syndrome and fatty liver: association or causal link?" World Journal of Gastroenterology, vol. 16, no. 34, pp. 4243-4252, 2010.

[2] M. H. Ahmed and C. D. Byrne, "Non alcoholic steatatohepatitis and metabolic syndrome," in Metabolic Syndrome, C. Byrne and S. Wild, Eds., pp. 279-305, John Wiley \& Sons, Chichester, UK, 2005.
[3] J. D. Browning, L. S. Szczepaniak, R. Dobbins et al., "Prevalence of hepatic steatosis in an urban population in the United States: impact of ethnicity," Hepatology, vol. 40, no. 6, pp. 1387-1395, 2004.

[4] L. S. Szczepaniak, P. Nurenberg, D. Leonard et al., "Magnetic resonance spectroscopy to measure hepatic triglyceride content: prevalence of hepatic steatosis in the general population," American Journal of Physiology, vol. 288, no. 2, pp. E462-E468, 2005.

[5] S. Bellentani, G. Saccoccio, F. Masutti et al., "Prevalence of and risk factors for hepatic steatosis in Northern Italy," Annals of Internal Medicine, vol. 132, no. 2, pp. 112-117, 2000.

[6] B. Liu, A. Balkwill, G. Reeves, and V. Beral, "Body mass index and risk of liver cirrhosis in middle aged UK women: prospective study," British Medical Journal, vol. 340, article c912, 2010.

[7] C. L. Hart, D. S. Morrison, G. D. Batty, R. J. Mitchell, and G. Davey Smith, "Effect of body mass index and alcohol consumption on liver disease: analysis of data from two prospective cohort studies," British Medical Journal, vol. 340, article c1240, 2010.

[8] M. H. Ahmed and C. D. Byrne, "Metabolic syndrome, diabetes \& CHD risk," in The Year in Lipid Disorders, C. J. Packard, Ed., pp. 3-26, Clinical Publishing, Oxford, UK, 2007.

[9] H. J. Kim, H. J. Kim, K. E. Lee et al., "Metabolic significance of nonalcoholic fatty liver disease in nonobese, nondiabetic adults," Archives of Internal Medicine, vol. 164, no. 19, pp. 2169-2175, 2004.

[10] J. B. Dixon, P. S. Bhathal, and P. E. O’Brien, "Nonalcoholic fatty liver disease: predictors of nonalcoholic steatohepatitis and liver fibrosis in the severely obese," Gastroenterology, vol. 121, no. 1, pp. 91-100, 2001.

[11] D. H. Akbar and A. H. Kawther, "Nonalcoholic fatty liver disease in Saudi type 2 diabetic subjects attending a medical outpatient clinic: prevalence and general characteristics," Diabetes Care, vol. 26, no. 12, pp. 3351-3352, 2003.

[12] S. I. Kojima, N. Watanabe, M. Numata, T. Ogawa, and S. Matsuzaki, "Increase in the prevalence of fatty liver in Japan over the past 12 years: analysis of clinical background," Journal of Gastroenterology, vol. 38, no. 10, pp. 954-961, 2003.

[13] J. G. Fan, J. Zhu, X. J. Li et al., "Fatty liver and the metabolic syndrome among Shanghai adults," Journal of Gastroenterology and Hepatology, vol. 20, no. 12, pp. 1825-1832, 2005.

[14] J. G. Fan, J. Zhu, X. J. Li et al., "Prevalence of and risk factors for fatty liver in a general population of Shanghai, China," Journal of Hepatology, vol. 43, no. 3, pp. 508-514, 2005.

[15] J. B. Schwimmer, R. Deutsch, T. Kahen, J. E. Lavine, C. Stanley, and C. Behling, "Prevalence of fatty liver in children and adolescents," Pediatrics, vol. 118, no. 4, pp. 1388-1393, 2006.

[16] G. Radetti, W. Kleon, J. Stuefer, and K. Pittschieler, "Nonalcoholic fatty liver disease in obese children evaluated by magnetic resonance imaging," Acta Paediatrica, vol. 95, no. 7, pp. 833-837, 2006.

[17] M. H. Ahmed, "Biochemical markers: the road map for the diagnosis of nonalcoholic fatty liver disease," American Journal of Clinical Pathology, vol. 127, no. 1, pp. 20-22, 2007.

[18] L. A. Adams, S. Harmsen, J. L. St Sauver et al., "Nonalcoholic fatty liver disease increases risk of death among patients with diabetes: a community-based cohort study," American Journal of Gastroenterology, vol. 105, no. 7, pp. 1567-1573, 2010.

[19] P. H. Chiang, T. Y. Chang, and J. D. Chen, "Synergistic effect of fatty liver and smoking on metabolic syndrome," World Journal of Gastroenterology, vol. 15, no. 42, pp. 5334-5339, 2009. 
[20] K. G. M. M. Alberti, R. H. Eckel, S. M. Grundy et al., "Harmonizing the metabolic syndrome: a joint interim statement of the international diabetes federation task force on epidemiology and prevention; National heart, lung, and blood institute; American heart association; World heart federation; International atherosclerosis society; and international association for the study of obesity," Circulation, vol. 120, no. 16, pp. 1640-1645, 2009.

[21] G. Bedogni, L. Miglioli, F. Masutti, C. Tiribelli, G. Marchesini, and S. Bellentani, "Prevalence of and risk factors for nonalcoholic fatty liver disease: the dionysos nutrition and liver study," Hepatology, vol. 42, no. 1, pp. 44-52, 2005.

[22] R. Rocha, H. P. Cotrim, F. M. Carvalho, A. C. Siqueira, H. Braga, and L. A. Freitas, "Body mass index and waist circumference in non-alcoholic fatty liver disease," Journal of Human Nutrition and Dietetics, vol. 18, no. 5, pp. 365-370, 2005.

[23] G. Pagano, G. Pacini, G. Musso et al., "Nonalcoholic steatohepatitis, insulin resistance, and metabolic syndrome: further evidence for an etiologic association," Hepatology, vol. 35, no. 2, pp. 367-372, 2002.

[24] R. Rocha, H. P. Cotrim, F. M. Carvalho, A. C. Siqueira, H. Braga, and L. A. Freitas, "Body mass index and waist circumference in non-alcoholic fatty liver disease," Journal of Human Nutrition and Dietetics, vol. 18, no. 5, pp. 365-370, 2005.

[25] S. Chitturi, S. Abeygunasekera, G. C. Farrell et al., "NASH and insulin resistance: insulinhypersecretion and specific association with the insulin resistance syndrome," Hepatology, vol. 35, no. 2, pp. 373-379, 2002.

[26] A. Gastaldelli, M. Kozakova, K. Höjlund et al., "Fatty liver is associated with insulin resistance, risk of coronary heart disease, and early atherosclerosis in a large European population," Hepatology, vol. 49, no. 5, pp. 1537-1544, 2009.

[27] S. Dam-Larsen, U. Becker, M. B. Franzmann, K. Larsen, P. Christoffersen, and F. Bendtsen, "Final results of a long-term, clinical follow-up in fatty liver patients," Scandinavian Journal of Gastroenterology, vol. 44, no. 10, pp. 1236-1243, 2009.

[28] N. Alkhouri, T. A. R. Tamimi, L. Yerian, R. Lopez, N. N. Zein, and A. E. Feldstein, "The inflamed liver and atherosclerosis: a Link between histologic severity of nonalcoholic fatty liver disease and increased cardiovascular risk," Digestive Diseases and Sciences, vol. 55, no. 9, pp. 2644-2650, 2010.

[29] J. P. Domanski, S. J. Park, and S. A. Harrison, "Cardiovascular disease and nonalcoholic fatty liver disease: does histologic severity matter?" Journal of Clinical Gastroenterology, vol. 46, no. 5, pp. 427-430, 2012.

[30] S. Ramilli, S. Pretolani, A. Muscari, B. Pacelli, and V. Arienti, "Carotid lesions in outpatients with nonalcoholic fatty liver disease," World Journal of Gastroenterology, vol. 15, no. 38, pp. 4770-4774, 2009.

[31] A. J. McCullough, "The clinical features, diagnosis and natural history of nonalcoholic fatty liver disease," Clinics in Liver Disease, vol. 8, no. 3, pp. 521-533, 2004.

[32] K. G. Tolman, V. Fonseca, M. H. Tan, and A. Dalpiaz, "Narrative review: hepatobiliary disease in type 2 diabetes mellitus," Annals of Internal Medicine, vol. 141, no. 12, pp. 946-956, 2004.

[33] P. Jousilahti, D. Rastenyte, and J. Tuomilehto, "Serum gammaglutamyl transferase, self-reported alcohol drinking, and the risk of stroke," Stroke, vol. 31, no. 8, pp. 1851-1855, 2000.

[34] G. Wannamethee, S. Ebrahim, and A. G. Shaper, "Gammaglutamyltransferase: determinants and association with mortality from ischemic heart disease and all causes," American Journal of Epidemiology, vol. 142, no. 7, pp. 699-708, 1995.
[35] M. Kozakova, C. Palombo, M. Paterni et al., "Fatty liver index, gamma-glutamyltransferase and early carotid plaques," Hepatology, vol. 55, no. 5, pp. 1406-1415, 2012.

[36] R. K. Schindhelm, J. M. Dekker, G. Nijpels et al., "Alanine aminotransferase predicts coronary heart disease events: a 10year follow-up of the Hoorn Study," Atherosclerosis, vol. 191, no. 2, pp. 391-396, 2007.

[37] C. C. Wang, S. K. Lin, Y. F. Tseng et al., "Elevation of serum aminotransferase activity increases risk of carotid atherosclerosis in patients with non-alcoholic fatty liver disease," Journal of Gastroenterology and Hepatology, vol. 24, no. 8, pp. 14111416, 2009.

[38] G. N. Ioannou, N. S. Weiss, E. J. Boyko, D. Mozaffarian, and S. P. Lee, "Elevated serum alanine aminotransferase activity and calculated risk of coronary heart disease in the United States," Hepatology, vol. 43, no. 5, pp. 1145-1151, 2006.

[39] M. Monami, G. Bardini, C. Lamanna et al., "Liver enzymes and risk of diabetes and cardiovascular disease: results of the Firenze Bagno a Ripoli (FIBAR) study," Metabolism, vol. 57, no. 3, pp. 387-392, 2008.

[40] A. Lopez-Suarez, J. M. Guerrero, J. Elvira-Gonzalez, M. Beltran-Robles, F. Canas-Hormigo, and A. Bascunana-Quirell, "Nonalcoholic fatty liver disease is associated with blood pressure in hypertensive and nonhypertensive individuals from the general population with normal levels of alanine aminotransferase," European Journal of Gastroenterology and Hepatology, vol. 23, no. 11, pp. 1011-1017, 2011.

[41] M. Lazo, R. Hernaez, S. Bonekamp et al., "Non-alcoholic fatty liver disease and mortality among US adults: prospective cohort study," British Medical Journal, vol. 343, article d6891, 2011.

[42] M. Stepanova and Z. M. Younossi, "Independent association between nonalcoholic fatty liver disease and cardiovascular disease in the US population," Clinical Gastroenterology and Hepatology, vol. 10, no. 6, pp. 646-650, 2012.

[43] G. Targher, L. Bertolini, R. Padovani et al., "Prevalence of nonalcoholic fatty liver disease and its association with cardiovascular disease among type 2 diabetic patients," Diabetes Care, vol. 30, no. 5, pp. 1212-1218, 2007.

[44] G. Targher, L. Bertolini, F. Poli et al., "Nonalcoholic fatty liver disease and risk of future cardiovascular events among type 2 diabetic patients," Diabetes, vol. 54, no. 12, pp. 3541-3546, 2005.

[45] L. Pacifico, V. Cantisani, P. Ricci et al., "Nonalcoholic fatty liver disease and carotid atherosclerosis in children," Pediatric Research, vol. 63, no. 4, pp. 423-427, 2008.

[46] J. B. Schwimmer, P. E. Pardee, J. E. Lavine, A. K. Blumkin, and S. Cook, "Cardiovascular risk factors and the metabolic syndrome in pediatric nonalcoholic fatty liver disease," Circulation, vol. 118, no. 3, pp. 277-283, 2008.

[47] A. L. Fracanzani, L. Burdick, S. Raselli et al., "Carotid artery intima-media thickness in nonalcoholic fatty liver disease," American Journal of Medicine, vol. 121, no. 1, pp. 72-78, 2008.

[48] G. Targher, L. Bertolini, R. Padovani et al., "Relations between carotid artery wall thickness and liver histology in subjects with nonalcoholic fatty liver disease," Diabetes Care, vol. 29, no. 6, pp. 1325-1330, 2006.

[49] H. Völzke, D. M. Robinson, V. Kleine et al., "Hepatic steatosis is associated with an increased risk of carotid atherosclerosis," World Journal of Gastroenterology, vol. 11, no. 12, pp. 18481853, 2005.

[50] J. M. Petit, B. Guiu, B. Terriat et al., "Nonalcoholic fatty liver is not associated with carotid intima-media thickness in 
type 2 diabetic patients," Journal of Clinical Endocrinology and Metabolism, vol. 94, no. 10, pp. 4103-4106, 2009.

[51] H. C. Kim, D. J. Kim, and K. B. Huh, "Association between nonalcoholic fatty liver disease and carotid intima-media thickness according to the presence of metabolic syndrome," Atherosclerosis, vol. 204, no. 2, pp. 521-525, 2009.

[52] R. K. Schindhelm, M. Diamant, S. J. L. Bakker et al., "Liver alanine aminotransferase, insulin resistance and endothelial dysfunction in normotriglyceridaemic subjects with type 2 diabetes mellitus," European Journal of Clinical Investigation, vol. 35, no. 6, pp. 369-374, 2005.

[53] N. Villanova, S. Moscatiello, S. Ramilli et al., "Endothelial dysfunction and cardiovascular risk profile in nonalcoholic fatty liver disease," Hepatology, vol. 42, no. 2, pp. 473-480, 2005.

[54] V. T. Samuel, Z. X. Liu, X. Qu et al., "Mechanism of hepatic insulin resistance in non-alcoholic fatty liver disease," Journal of Biological Chemistry, vol. 279, no. 31, pp. 32345-32353, 2004.

[55] D. Cai, M. Yuan, D. F. Frantz et al., "Local and systemic insulin resistance resulting from hepatic activation of IKK- $\beta$ and NF$\kappa \mathrm{B}$, , Nature Medicine, vol. 11, no. 2, pp. 183-190, 2005.

[56] G. Musso, R. Gambino, F. De Michieli, M. Durazzo, G. Pagano, and M. Cassader, "Adiponectin gene polymorphisms modulate acute adiponectin response to dietary fat: possible pathogenetic role in NASH," Hepatology, vol. 47, no. 4, pp. 1167-1177, 2008.

[57] C. Pagano, G. Soardo, W. Esposito et al., "Plasma adiponectin is decreased in nonalcoholic fatty liver disease," European Journal of Endocrinology, vol. 152, no. 1, pp. 113-118, 2005.

[58] G. Targher, L. Bertolini, L. Scala, F. Poli, L. Zenari, and G. Falezza, "Decreased plasma adiponectin concentrations are closely associated with nonalcoholic hepatic steatosis in obese individuals," Clinical Endocrinology, vol. 61, no. 6, pp. 700703, 2004.

[59] T. Pischon, C. J. Girman, G. S. Hotamisligil, N. Rifai, F. B. $\mathrm{Hu}$, and E. B. Rimm, "Plasma adiponectin levels and risk of myocardial infarction in Men," Journal of the American Medical Association, vol. 291, no. 14, pp. 1730-1737, 2004.

[60] N. Matikainen, S. Mänttäri, J. Westerbacka et al., "Postprandial lipemia associates with liver fat content," Journal of Clinical Endocrinology and Metabolism, vol. 92, no. 8, pp. 3052-3059, 2007.

[61] H. Basciano, A. E. Miller, M. Naples et al., "Metabolic effects of dietary cholesterol in an animal model of insulin resistance and hepatic steatosis," American Journal of Physiology, vol. 297, no. 2, pp. E462-E473, 2009.

[62] F. Hadaegh, H. Harati, A. Ghanbarian, and F. Azizi, "Prevalence of coronary heart disease among Tehran adults: Tehran Lipid and Glucose Study," Eastern Mediterranean Health Journal, vol. 15, no. 1, pp. 157-166, 2009.

[63] B. G. Nordestgaard, M. Benn, P. Schnohr, and A. TybjaergHansen, "Nonfasting triglycerides and risk of myocardial infarction, ischemic heart disease, and death in men and women," Journal of the American Medical Association, vol. 298, no. 3, pp. 299-308, 2007.

[64] S. Bansal, J. E. Buring, N. Rifai, S. Mora, F. M. Sacks, and P. M. Ridker, "Fasting compared with nonfasting triglycerides and risk of cardiovascular events in women," Journal of the American Medical Association, vol. 298, no. 3, pp. 309-316, 2007.

[65] J. J. Freiberg, A. Tybjærg-Hansen, J. S. Jensen, and B. G. Nordestgaard, "Nonfasting triglycerides and risk of ischemic stroke in the general population," Journal of the American Medical Association, vol. 300, no. 18, pp. 2142-2152, 2008.

[66] S. Mora, N. Rifai, J. E. Buring, and P. M. Ridker, "Fasting compared with nonfasting lipids and apolipoproteins for predicting incident cardiovascular events," Circulation, vol. 118, no. 10, pp. 993-1001, 2008.

[67] D. Kim, S. Y. Choi, E. H. Park et al., "Nonalcoholic fatty liver disease is associated with coronary artery calcification," Hepatology, vol. 56, no. 2, pp. 605-613, 2012.

[68] Y. Yilmaz, R. Kurt, O. Yonal et al., "Coronary flow reserve is impaired in patients with nonalcoholic fatty liver disease: association with liver fibrosis," Atherosclerosis, vol. 211, no. 1, pp. 182-186, 2010.

[69] S. Goland, S. Shimoni, T. Zornitzki et al., "Cardiac abnormalities as a new manifestation of nonalcoholic fatty liver disease: echocardiographic and tissue Doppler imaging assessment," Journal of Clinical Gastroenterology, vol. 40, no. 10, pp. 949 955, 2006

[70] S. Bonapace, G. Perseghin, G. Molon et al., "Nonalcoholic fatty liver disease is associated with left ventricular diastolic dysfunction in patients with type 2 diabetes," Diabetes Care, vol. 35, no. 2, pp. 389-395, 2012.

[71] T. Young, M. Palta, J. Dempsey, J. Skatrud, S. Weber, and S. Badr, "The occurrence of sleep-disordered breathing among middle-aged adults," The New England Journal of Medicine, vol. 328, no. 17, pp. 1230-1235, 1993.

[72] W. W. Flemons, D. Buysse, S. Redline et al., "Sleep-related breathing disorders in adults: recommendations for syndrome definition and measurement techniques in clinical research. The Report of an American Academy of Sleep Medicine Task Force," Sleep, vol. 22, no. 5, pp. 667-689, 1999.

[73] C. D. Byrne, "Hypoxia and non-alcoholic fatty liver disease," Clinical Science, vol. 118, no. 6, pp. 397-400, 2010.

[74] M. Nishibayashi, M. Miyamoto, T. Miyamoto, K. Suzuki, and K. Hirata, "Correlation between severity of obstructive sleep apnea and prevalence of silent cerebrovascular lesions," Journal of Clinical Sleep Medicine, vol. 4, no. 3, pp. 242-247, 2008.

[75] Z. Dorkova, D. Petrasova, A. Molcanyiova, M. Popovnakova, and R. Tkacova, "Effects of continuous positive airway pressure on cardiovascular risk profile in patients with severe obstructive sleep apnea and metabolic syndrome," Chest, vol. 134, no. 4, pp. 686-692, 2008.

[76] N. Takama and M. Kurabayashi, "Influence of untreated sleepdisordered breathing on the long-term prognosis of patients with cardiovascular disease," American Journal of Cardiology, vol. 103, no. 5, pp. 730-734, 2009.

[77] V. Savransky, A. Nanayakkara, A. Vivero et al., "Chronic intermittent hypoxia predisposes to liver injury," Hepatology, vol. 45, no. 4, pp. 1007-1013, 2007.

[78] V. Savransky, S. Bevans, A. Nanayakkara et al., "Chronic intermittent hypoxia causes hepatitis in a mouse model of diet-induced fatty liver," American Journal of Physiology, vol. 293, no. 4, pp. G871-G877, 2007.

[79] F. Takayama, T. Egashira, H. Kawasaki et al., "A novel animal model of nonalcoholic steatohepatitis (NASH): hypoxemia enhances the development of NASH," Journal of Clinical Biochemistry and Nutrition, vol. 45, no. 3, pp. 335-340, 2009.

[80] A. C. Piguet, D. Stroka, A. Zimmermann, and J. F. Dufour, "Hypoxia aggravates non-alcoholic steatohepatitis in mice lacking hepatocellular PTEN," Clinical Science, vol. 118, no. 6, pp. 401-410, 2010. 
[81] T. D. Bradley and J. S. Floras, "Obstructive sleep apnoea and its cardiovascular consequences," The Lancet, vol. 373, no. 9657, pp. 82-93, 2009.

[82] E. C. Fletcher, R. D. DeBehnke, M. S. Lovoi, and A. B. Gorin, "Undiagnosed sleep apnea in patients with essential hypertension," Annals of Internal Medicine, vol. 103, no. 2, pp. 190195, 1985.

[83] A. G. Logan, S. M. Perlikowski, A. Mente et al., "High prevalence of unrecognized sleep apnoea in drug-resistant hypertension," Journal of Hypertension, vol. 19, no. 12, pp. 22712277, 2001.

[84] J. Börgel, B. M. Sanner, F. Keskin et al., "Obstructive sleep apnea and blood pressure: interaction between the blood pressure-lowering effects of positive airway pressure therapy and antihypertensive drugs," American Journal of Hypertension, vol. 17, no. 12, pp. 1081-1087, 2004.

[85] Y. Peker, H. Kraiczi, J. Hedner, S. Löth, A. Johansson, and M. Bende, "An independent association between obstructive sleep apnoea and coronary artery disease," European Respiratory Journal, vol. 14, no. 1, pp. 179-184, 1999.

[86] T. Mooe, K. A. Franklin, K. Holmström, T. Rabben, and U. Wiklund, "Sleep-disordered breathing and coronary artery disease: long-term prognosis," American Journal of Respiratory and Critical Care Medicine, vol. 164, no. 10, pp. 1910-1913, 2001.

[87] D. C. Good, J. Q. Henkle, D. Gelber, J. Welsh, and S. Verhulst, "Sleep-disordered breathing and poor functional outcome after stroke," Stroke, vol. 27, no. 2, pp. 252-259, 1996.

[88] Y. Kaneko, V. E. Hajek, V. Zivanovic, J. Raboud, and T. D. Bradley, "Relationship of sleep apnea to functional capacity and length of hospitalization following stroke," Sleep, vol. 26, no. 3, pp. 293-297, 2003.

[89] G. Targher, L. Bertolini, S. Rodella et al., "Non-alcoholic fatty liver disease is independently associated with an increased prevalence of chronic kidney disease and proliferative/lasertreated retinopathy in type 2 diabetic patients," Diabetologia, vol. 51, no. 3, pp. 444-450, 2008.

[90] G. Targher, M. Chonchol, L. Bertolini et al., "Increased risk of CKD among type 2 diabetics with nonalcoholic fatty liver disease," Journal of the American Society of Nephrology, vol. 19, no. 8, pp. 1564-1570, 2008.

[91] Y. Chang, S. Ryu, E. Sung et al., "Nonalcoholic fatty liver disease predicts chronic kidney disease in nonhypertensive and nondiabetic Korean men," Metabolism, vol. 57, no. 4, pp. 569-576, 2008.

[92] N. Chalasani, Z. Younossi, J. E. Lavine et al., "The diagnosis and management of non-alcoholic fatty liver disease: practice Guideline by the American Association for the Study of Liver Diseases, American College of Gastroenterology, and the American Gastroenterological Association," Hepatology, vol. 55, no. 6, pp. 2005-2023, 2012.

[93] D. E. Cohen, F. A. Anania, and N. Chalasani, "An assessment of statin safety by hepatologists," American Journal of Cardiology, vol. 97, no. 8, pp. S77-S81, 2006.

[94] M. H. Ahmed and C. D. Byrne, "Current treatment of nonalcoholic fatty liver disease," Diabetes, Obesity and Metabolism, vol. 11, no. 3, pp. 188-195, 2009. 


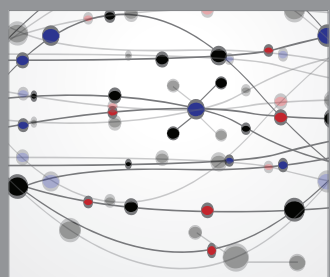

The Scientific World Journal
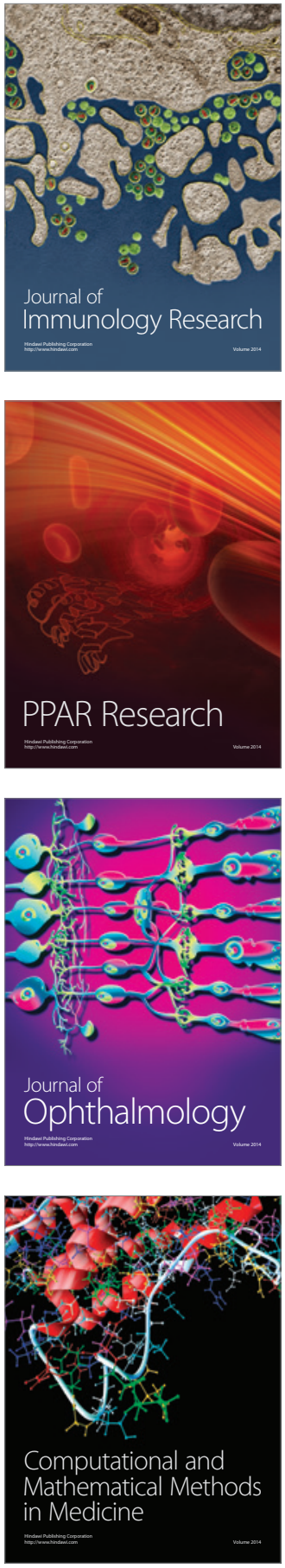

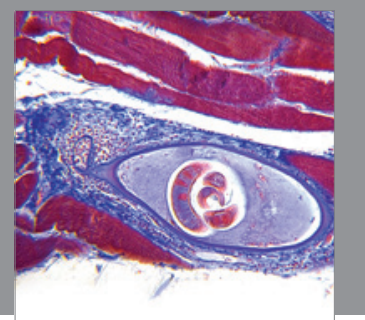

Gastroenterology

Research and Practice
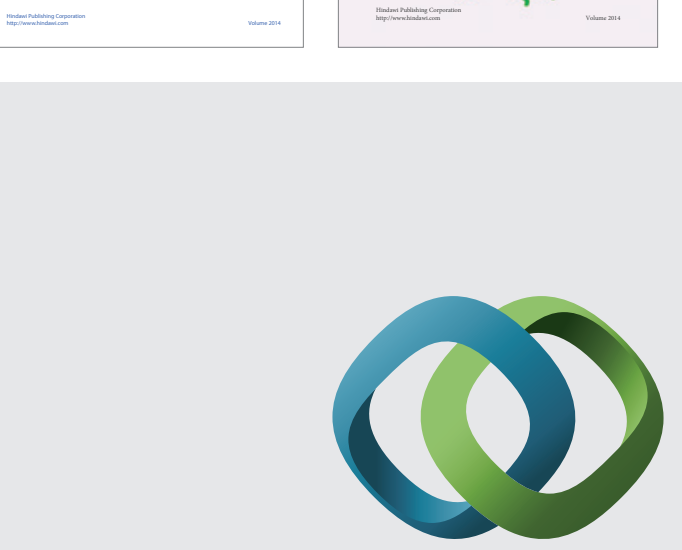

\section{Hindawi}

Submit your manuscripts at

http://www.hindawi.com
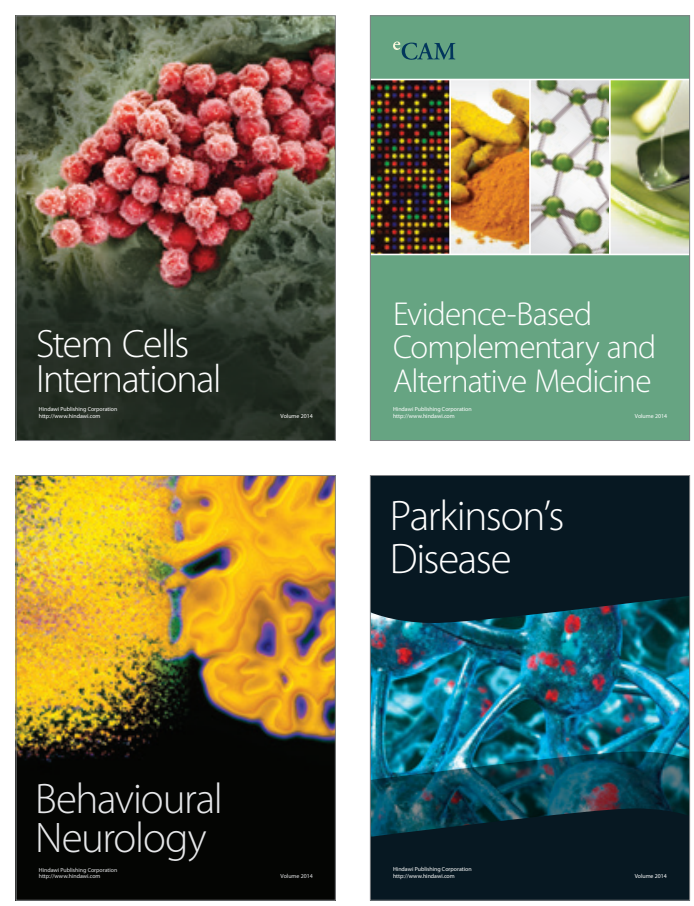

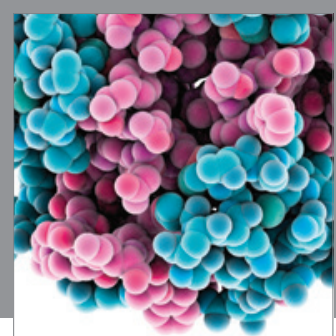

Journal of
Diabetes Research

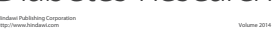

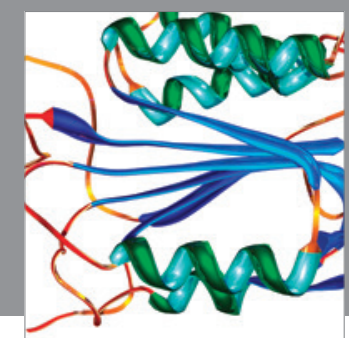

Disease Markers
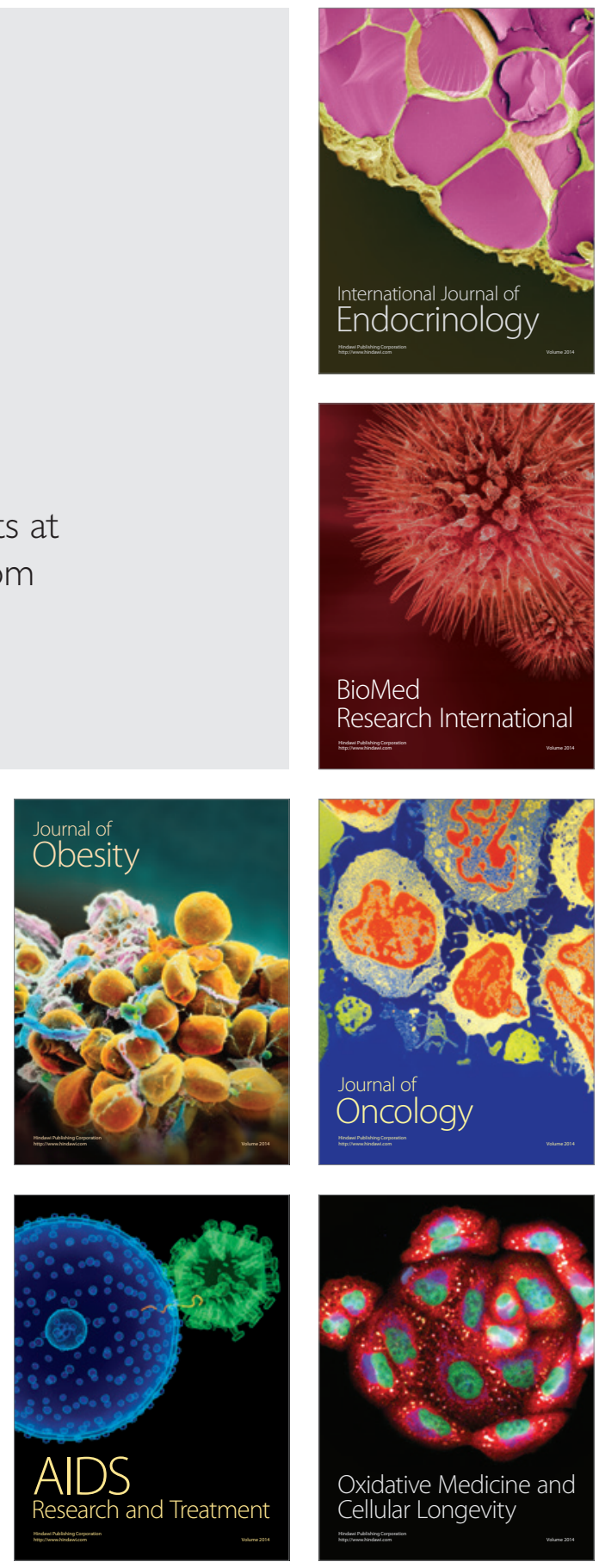